\title{
LA FASE ESPONJA COMO SISTEMA BIOMIMÉTICO PARA CRISTALIZAR PROTEÍNAS DE MEMBRANA
}

\author{
The sponge phase as biomimetic system \\ for crystallizing membrane protein
}

\section{EPISTEMUS}

ISSN: 2007-8196 (electrónico)

ISSN: 2007-4530 (impresa)

Amir Darío Maldonado Arce 1

Ramón Íñiguez Palomares 2

Ricardo López Esparza 3

Gerardo Paredes Quijada 4

Judith Tánori Córdova ${ }^{5}$

Recibido: 18 de septiembre de 2015,

Aceptado: 22 de febrero del 2016

Autor de Correspondencia:

Dr. Amir Maldonado Arce

Correo: maldona@guaymas.uson.mx

\section{Resumen}

En este trabajo se describe de manera general la importancia de la cristalización de proteínas para realizar experimentos de difracción de rayos $X$ que permitan dilucidar la estructura terciaria de dichas biomoléculas. En particular, se expone que la cristalización de proteínas de membrana requiere métodos especiales de preparación de la matriz de cristalización puesto que se debe "mimetizar" el ambiente hidrofóbico de la proteína en la membrana. De esta manera, el trabajo se centra en algunas propiedades de la fase líquida de membranas denominada "fase esponja", cuya característica principal es una estructura membranar compleja conectada en tres dimensiones. Debido a su microestructura, la fase esponja es transparente e isotrópica, además de presentar baja viscosidad en las membranas. Estas características convierten a la fase esponja en un medio "biomimético" potencialmente útil para cristalizar proteínas de membrana.

Palabras clave: Fase esponja, biomimética, cristalización, proteínas.

\begin{abstract}
This paper describes in general terms the importance of protein crystallization and $X$-ray diffraction experiments used to elucidate the tertiary structure of these biomolecules. In particular, it is stated that crystallization of membrane proteins requires special methods of preparation of the crystallization matrix since it must "mimic" the hydrophobic environment of the membrane protein. Thus, the work focuses on some properties of the liquid phase of membranes called "sponge phase", whose main feature is a complex structure of membranes connected in three dimensions. Due to their microstructure, the sponge is a transparent, isotropic liquid that have a low viscosity. These features make the sponge phase a "biomimetic" system potentially useful for crystallizing membrane proteins.
\end{abstract}

1 Departamento de Física, Universidad de Sonora / Correo: maldona@guaymas.uson.mx 


\section{INTRODUCCIÓN}

Un problema central de la biología molecular es la determinación de la estructura tridimensional de proteínas. Esto se debe a que la geometría de dichas moléculas está estrechamente relacionada con su función biológica.

La caracterización estructural de proteínas se lleva a cabo principalmente mediante la técnica de difracción de rayos $\mathrm{X}$, la cual tiene como requisito previo la obtención de cristales macroscópicos de la molécula bajo estudio. Por esta razón, el desarrollo de métodos de obtención de cristales de proteína es un tema científico que presenta gran interés en la actualidad.

En este documento se comentan algunas características del proceso de cristalización de un tipo particular de proteínas: las de membrana. Así mismo, se discuten algunas propiedades físicas relevantes de la "fase esponja", la cual es objeto de estudio como alternativa biomimética para cristalizar proteínas de membrana en un ambiente físico relativamente próximo a una membrana biológica. Biomimética en este sentido, es la "imitación" que hace una membrana modelo (artificial, creada en el laboratorio) de las propiedades de la membrana celular [1]. En la figura 1 se ilustran algunas de estas propiedades.

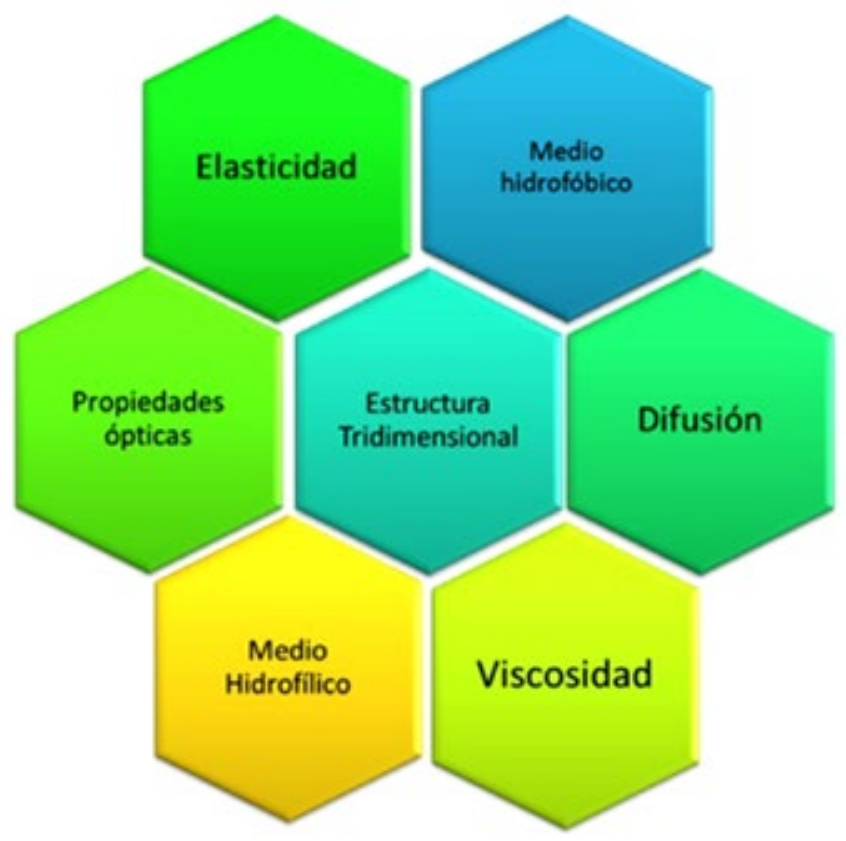

Figura 1. Algunas propiedades de la membrana celular que pueden "imitarse" por membranas artificiales.

\section{CRISTALIZACIÓN DE PROTEÍNAS DE MEMBRANA}

Un cristal de proteínas es un arreglo periódico tridimensional de dichas moléculas. Cuando un haz de rayos $X$ incide sobre el cristal, la periodicidad ocasiona que la difracción de las ondas electromagnéticas ocurra de una manera específica: para ciertos ángulos, la interferencia constructiva de los haces difractados en diferentes capas del cristal resulta en máximos de difracción o "picos de Bragg". De la posición angular de los picos en un patrón de difracción, es posible deducir las diferentes distancias características del cristal, y por lo tanto, la posición de cada átomo en la proteína. De esta manera se han podido determinar las estructuras tridimensionales de proteínas con una resolución hasta de unos pocos Angstroms [2,3].

A manera de ejemplo, en la figura 2 se presenta un esquema de la estructura terciaria de la mioglobina, junto con un cristal de la misma proteína. La mayoría de las estructuras de proteínas reportadas se han determinado por difracción de rayos X. Según el Protein Data Bank, en 2010, el $86.7 \%$ de las estructuras se han obtenido por este método; a comparación con el $13 \%$ por Resonancia Magnética Nuclear (RMN) y menos del $1 \%$ por microscopía electrónica [4].

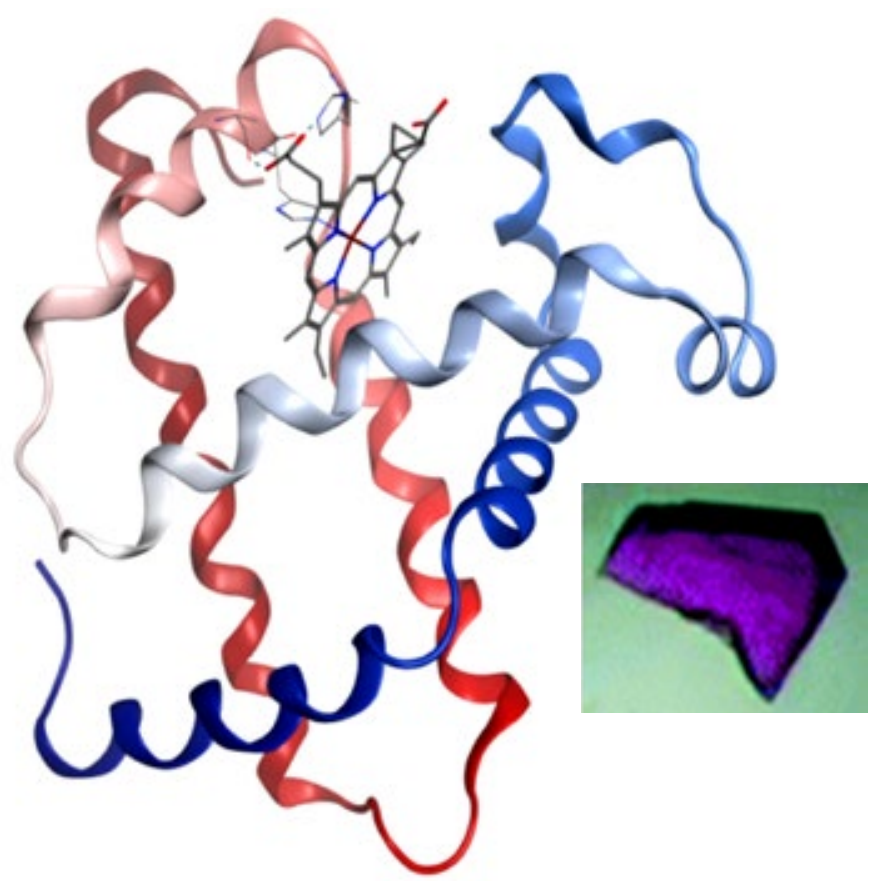

Figura 2. Representación de la estructura terciaria de la mioglobina y cristal de dicha proteína.

Dada la importancia de los experimentos de difracción de rayos $X$, la cristalografía de proteínas es un área que recibe mucha atención: donde se buscan métodos que permitan preparar cristales de proteínas de interés.

En el caso de las proteínas hidrosolubles, dichos métodos parten de soluciones de proteína en medios fisiológicos. Sin embargo, el caso de las proteínas de membrana es más complejo puesto que se trata de moléculas insolubles en agua; en el estado natural, estas proteínas se encuentran incorporadas a la matriz fosfolipídica de las membranas biológicas. Por esta razón, la cristalización de estas moléculas requiere un medio para "mimetizar" el ambiente hidrofóbico de las membranas y permitir que las proteínas adquieran su conformación 
nativa. Se estima que las proteínas de membrana representan entre el 20 y el $30 \%$ del total de proteínas del ser humano.

Un método empleado para cristalizar este tipo de proteínas consiste en su incorporación en micelas de surfactante, donde se induce la formación del cristal. En la figura 3a se muestra un esquema de la cristalización de proteínas en micelas. Sin embargo, este procedimiento tiene la desventaja de que las micelas presentan una geometría muy diferente que la de una membrana biológica y por lo tanto la proteína no tiene un medio ambiente similar al natural. Esto significa que la estructura que se determina a partir de los cristales preparados en micelas no necesariamente corresponde a la estructura nativa de las proteínas [5]. Por esta razón es importante encontrar nuevos medios "biomiméticos" que permitan cristalizar proteínas de membrana en ambientes más cercanos al natural, tal como se aprecia en la figura 3b. La llamada "fase esponja" es un medio líquido que tiene propiedades interesantes para este fin; a continuación se describe con más detalle.

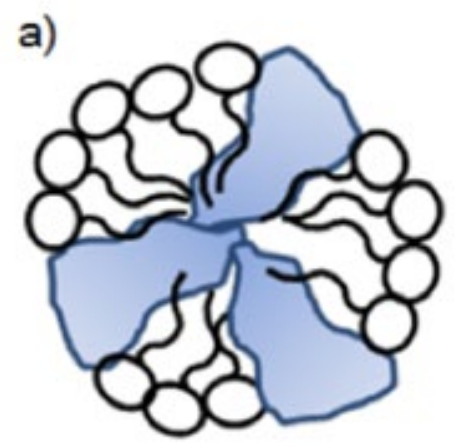

b)

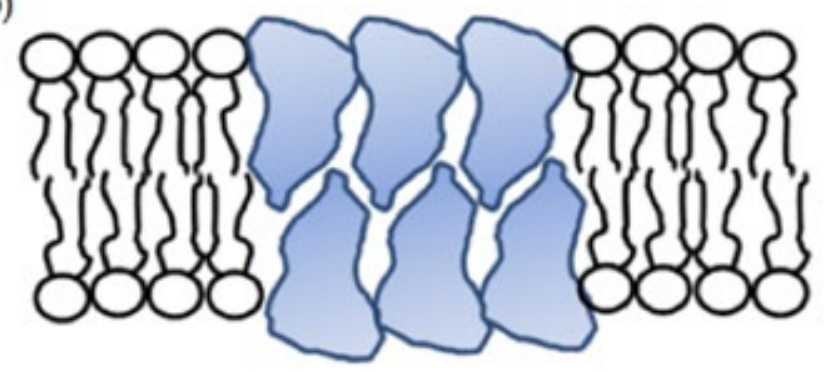

Figura 3. Esquema de la cristalización de proteínas en (a) micelas de surfactante y (b) membranas de una fase esponja de fosfolípido.

\section{LA FASE ESPONJA}

Se denomina fase esponja a un sistema líquido formado por membranas en un solvente. Las membranas pueden ser de un surfactante como el Dodecil sulfato de sodio (SDS) o de un fosfolípido como la fosfatidilcolina. El solvente puede ser agua pura o alguna solución fisiológica.

Los surfactantes y los fosfolípidos están constituidos por moléculas anfifílicas, las cuales tienen un grupo polar (soluble en agua) y un grupo hidrofóbico (insoluble en agua). Al tratar de solubilizar estos materiales en agua, las moléculas se agrupan de tal forma que su parte hidrofóbica evita el contacto con el solvente. Este es el principio de formación de las micelas y de las membranas (Figura 3).

Dependiendo de las condiciones fisicoquímicas del sistema (concentración de surfactante o de fosfolípido, temperatura, salinidad, etc.) las membranas formadas pueden acomodarse en diferentes geometrías. La más simple es la denominada "fase lamelar", donde las membranas son planas y están ordenadas de manera periódica, de forma similar a las hojas de un libro; en el espacio entre membranas se encuentra el solvente. Otra geometría usual es la de "vesículas" o "liposomas"; en ella, la membrana se cierra sobre sí misma, generalmente en una forma esférica, y encapsula un pequeño volumen de solvente. Finalmente, en la fase esponja, las membranas se conectan en una geometría tridimensional compleja.

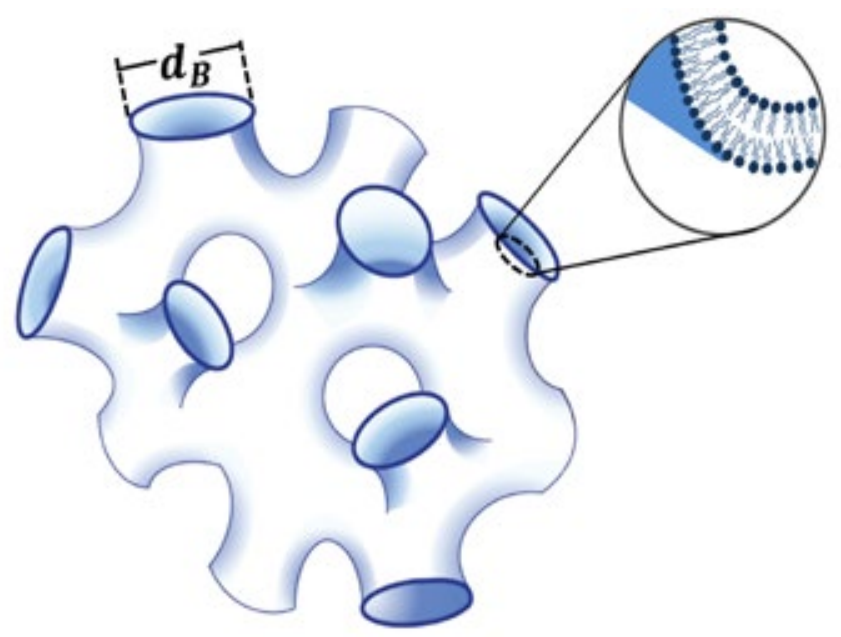

Figura 4. Representación esquemática de una fase esponja.

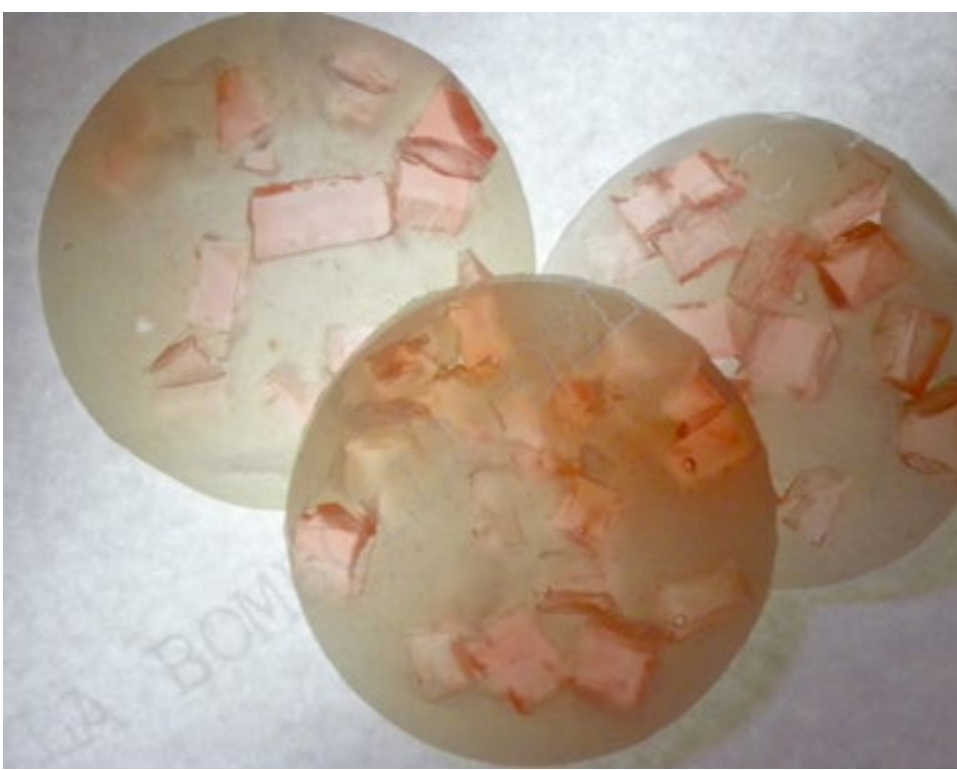


En la figura 4 se muestra un dibujo esquemático de una fase esponja. La estructura observada representa una membrana conectada en todo el espacio de la muestra, la cual forma poros o canales, dentro y fuera de los cuales está el solvente. Dada la similitud con un medio poroso, como una esponja, el líquido recibe este nombre: "fase esponja". También se le conoce como fase L3. La fase esponja guarda cierta similitud con una fase cúbica de membranas, con la diferencia de que no presenta un arreglo periódico de bicapas. Por esta razón, es común llamarle también "fase cúbica fundida". En la figura 5 se presenta una imagen microscópica de una fase esponja obtenida por microscopía electrónica de criofractura.

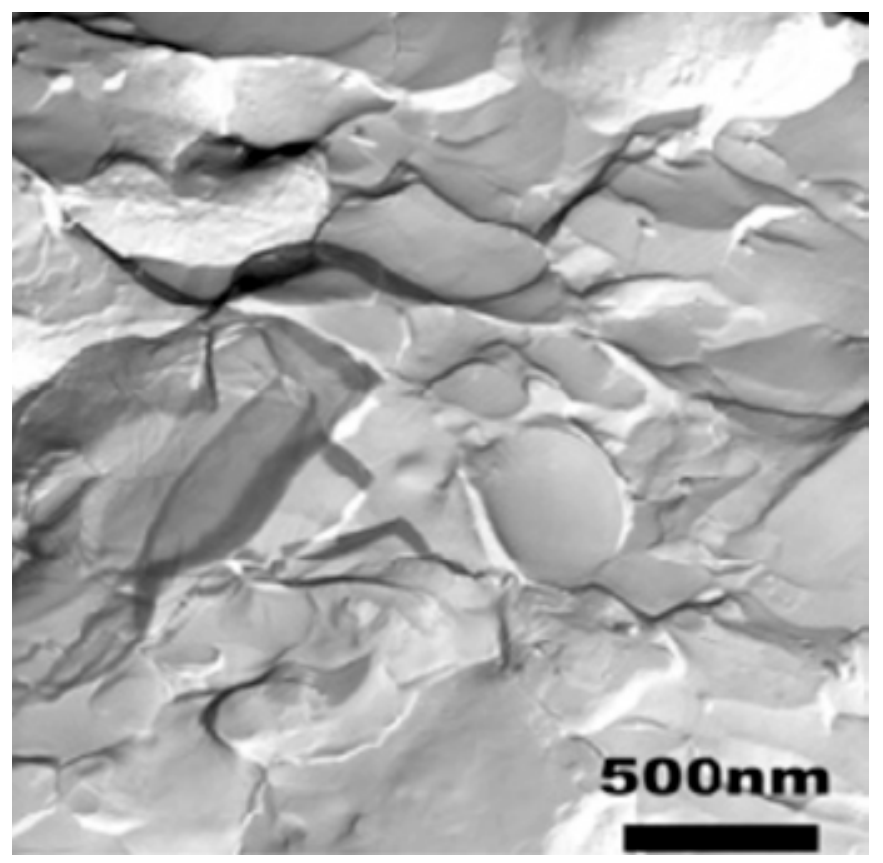

Figura 5. Fotografía obtenida por microscopia electrónica de criofractura de una fase esponja del sistema SDS-hexanol-solución salina.

La fase esponja ha sido observada en algunos sistemas de surfactante y de fosfolípido. Entre tales sistemas se encuentran surfactantes iónicos como el SDS, neutros como la familia de los EiCj e incluso zwitteriónicos como el C14DMAO $[6,7,8]$.

Para cada sistema, esta fase aparece en una región definida del diagrama de fases, muchas veces en la vecindad de una fase lamelar de membranas. Usualmente, la adición de un cosurfactante, como un alcohol de cadena corta, o incluso la variación en la concentración salina, induce la aparición de la fase esponja. A manera de ejemplo, en la figura 6 se muestra el diagrama de fases del sistema monooleina-agua-PEG; en este caso, las fase esponja es inducida al agregar el polímero polietilenglicol (PEG) a una solución del lípido monooleína. Este sistema ha recibido mucha atención por la posibilidad de utilizarlo para cristalizar proteínas de membrana [10].

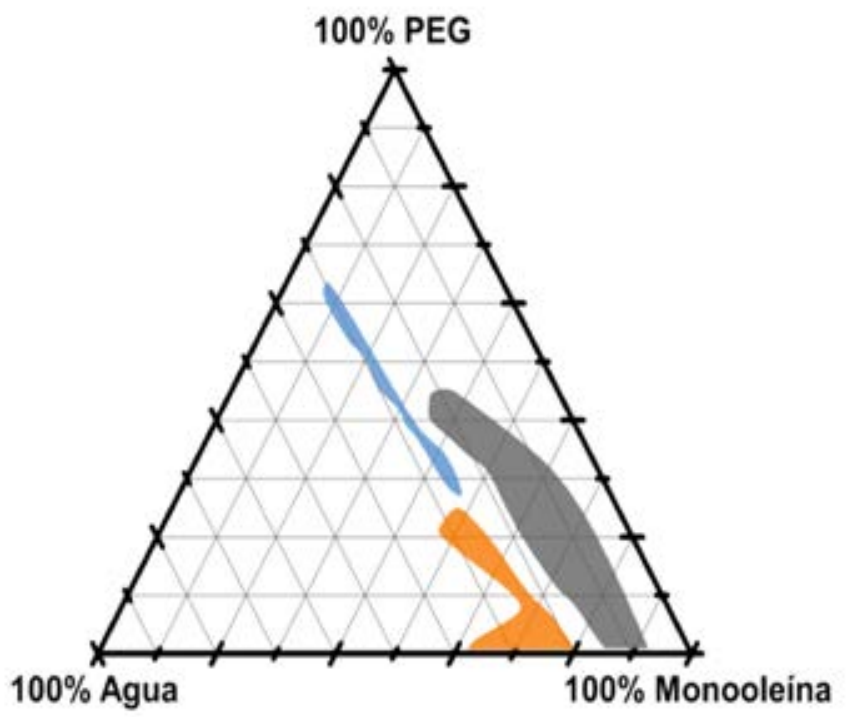

Figura 6. Diagrama de fases parcial del sistema monooleína-agua-PEG. La región azul corresponde a la fase esponja, la naranja a la fase cúbica y la gris a la fase lamelar (Adaptada de la referencia 9). ALGUNAS PROPIEDADES DE LA FASE ESPONJA

La fase esponja presenta propiedades físicas muy particulares si se le compara con otros sistemas de membranas (lamelares, cúbicas, vesiculares). A continuación mencionamos algunas de dichas propiedades que se originan en su estructura tridimensional compleja.

\section{Propiedades ópticas}

En primer término, las fases esponja es transparente a simple vista, lo cual permite visualizar por medios ópticos tradicionales la presencia de estructuras inmersas en ella, tales como eventuales cristales de proteína. Además, dado que la fase es desordenada, las membranas no presentan ninguna dirección espacial privilegiada y por lo tanto no son birrefringentes. Esta isotropía, facilita la observación por medio de la microscopía óptica de luz polarizada de estructuras presentes en su seno, que si tienen birrefringencia.

\section{Viscosidad}

Otra propiedad interesante de la fase es su baja viscosidad, la cual es más próxima a la del agua que la viscosidad que presentan otras fases de membrana como la cúbica y lamelar; esto a pesar de que la concentración de membranas es similar en las tres fases mencionadas. Esta baja viscosidad se debe a que las membranas están conectadas de manera desordenada en las tres dimensiones del espacio. En consecuencia, la fase esponja es fácil de manipular, con poca resistencia al flujo, permite disolver aditivos (como proteínas) fácilmente e inhibe la formación de burbujas de aire en su interior. 


\section{Características estructurales}

A pesar de que el arreglo espacial de las membranas de una fase esponja es desordenado, existe una distancia (dB), característica del sistema: el tamaño de los poros o equivalentemente la distancia media entre membranas (señalada en la figura $\mathrm{X}$ ). La distancia $\mathrm{dB}$ para la fase esponja es del orden de 10 a $100 \mathrm{~nm}$ dependiendo de la concentración y tipo de surfactante utilizado en su elaboración. Para determinar su valor, se utilizan técnicas sofisticadas, entre las cuales se tiene la dispersión de rayos $\mathrm{X}$ a ángulos pequeños (SAXS por sus siglas en inglés).

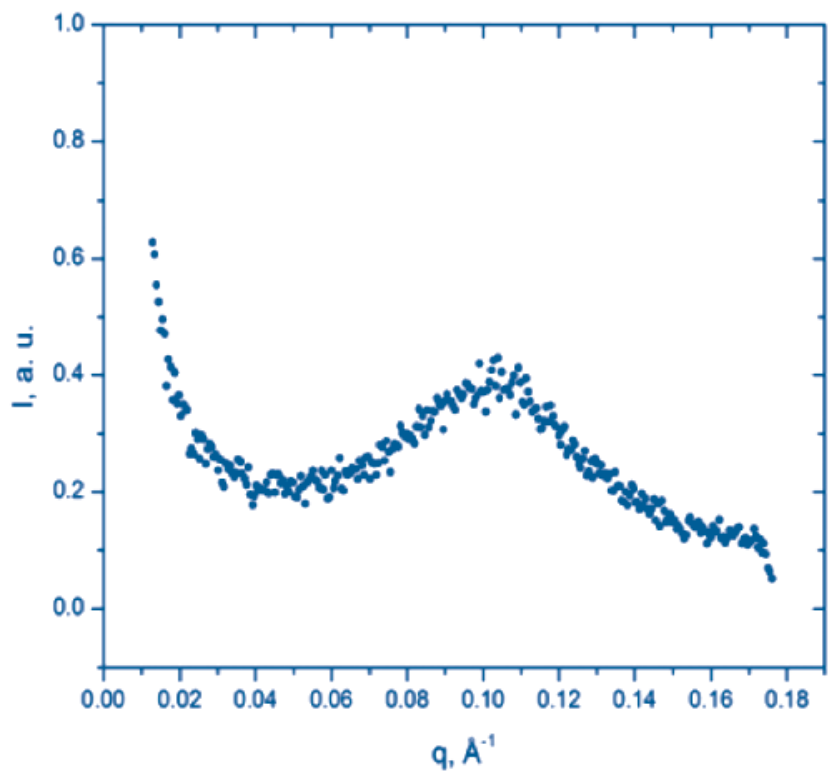

En la figura 7 se muestra un espectro de dispersión obtenido con esta técnica para una fase esponja; el eje vertical representa la intensidad de dispersión normalizada, mientras que el eje horizontal es el vector de onda, relacionado al ángulo de dispersión y es la longitud de onda de los rayos $\mathrm{X}$. De la posición del máximo en la gráfica (q del máximo o qmax) se obtiene $\mathrm{dB}$ mediante la ecuación de Bragg: . A manera de comparación, en la misma figura también se presenta un espectro de SAXS de una fase lamelar; se observa que en este caso el pico máximo es más pronunciado y estrecho que en la fase esponja, lo que implica mayor orden espacial de las membranas.

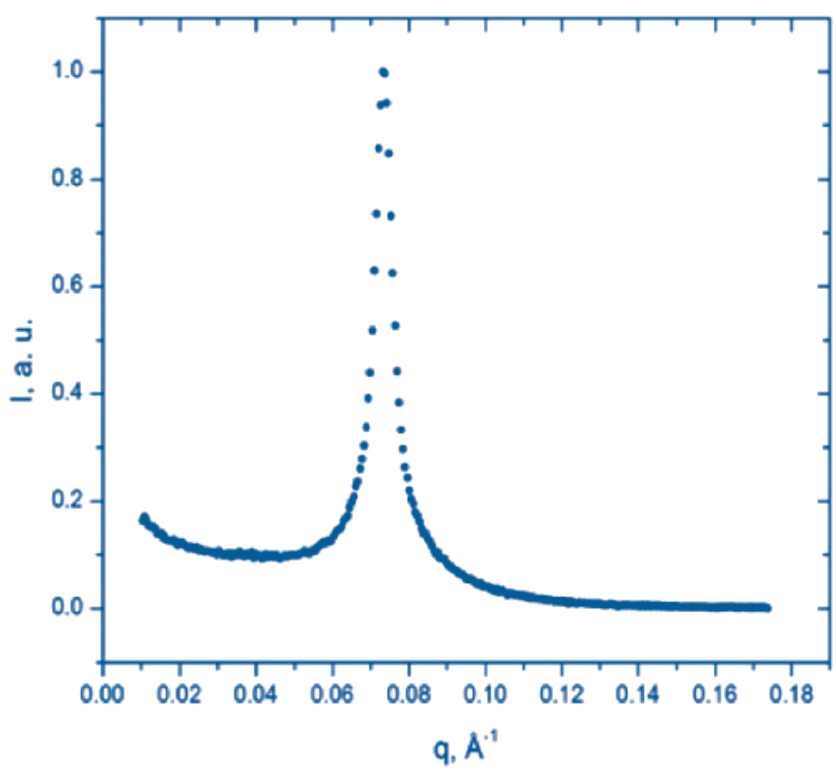

Figura 7. Espectros de SAXS para una fase esponja (izquierda) y una fase lamelar (derecha) (Espectros obtenidos por el Dr. Ricardo López Esparza en el Collège de France).

\section{Difusión en la fase esponja}

Finalmente, una propiedad muy importante de la fase esponja es la fluidez de sus membranas, que permiten la difusión de moléculas confinadas en ellas. Por ejemplo, si se solubiliza alguna molécula con un grupo hidrofóbico, en estas membranas, es posible seguir su difusión mediante técnicas como la recuperación de fluorescencia después del fotoblanqueado (FRAP). De esta forma se ha medido el coeficiente de difusión de proteínas, péptidos [11] y moléculas de fosfolípido [12].

A manera de ilustración, en la figura 8 se presenta una gráfica del coeficiente de difusión de un péptido y una molécula de surfactante, medidos con FRAP. La movilidad de moléculas en la membrana es esencial para la cristalización de proteínas, puesto que estas moléculas deben desplazarse sobre la membrana para organizarse espontáneamente en función de las fuerzas intermoleculares de atracción.

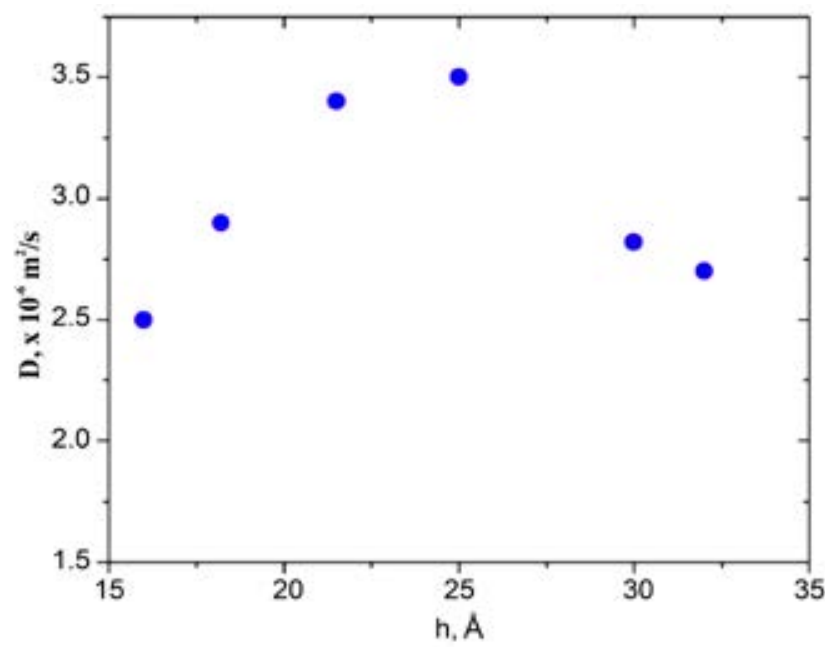

Figura 8. Coeficientes de difusion de un péptido transmembranar función del espesor de membrana $h$ de una fase esponja ternaria del sistema C12E5-agua-ßOG. 


\section{LA FASE ESPONJA COMO MEDIO BIOMIMÉTICO}

Las propiedades mencionadas permiten concebir a la fase esponja como un medio biomimético para inducir la cristalización de proteínas de membrana. El ambiente hidrofóbico que proporciona la bicapa de lípido o de surfactante es similar al que tienen las proteínas en la célula. Aunado a esto se suman la transparencia, la isotropía, la baja viscosidad y la fluidez de la membrana, propiedades apropiadas para seguir el proceso de cristalización [13-18].

Estas características hacen interesante el estudio de la influencia de la incorporación de proteínas sobre la propia fase esponja. Por ejemplo, antes de cristalizar una proteína, es necesario caracterizar el diagrama de fases del sistema lípido-proteína, pues es posible que la adición de la biomolécula provoque desplazamientos en el diagrama de fases y/o modificación de las propiedades físicas del sistema. Esta caracterización puede lograrse mediante experimentos de SAXS o microscopía electrónica de criofractura. Otras propiedades, como el coeficiente de difusión de las proteínas, son importantes puesto que de su magnitud depende la facilidad con la que las proteínas se mueven en la membrana en el proceso de atracción que lleva a la cristalización.

Finalmente, el estudio teórico de sistemas proteínafase esponja es necesario puesto que es indispensable entender los mecanismos moleculares que llevan a la formación de un cristal de proteínas. Estos mecanismos dependen de las fuerzas de interacción, las cuales pueden tener particularidades según el tipo de proteína y de membrana (neutra, cargada, etc). Conociendo los mecanismos moleculares, en principio se pueden buscar condiciones óptimas de cristalización al variar parámetros como la salinidad (apantallamiento de cargas) o la concentración de aditivos como polímeros.

\section{CONCLUSIONES}

En este trabajo se ha descrito brevemente el problema de la cristalización de proteínas de membrana. Así mismo, se han comentado algunas propiedades de la llamada fase esponja, sistema líquido que se está estudiando actualmente por varios grupos de investigación con el objetivo de cristalizar proteínas. Es factible que en un futuro próximo este sistema desplace a las micelas de surfactante como soporte para la obtención de cristales apropiados para experimentos de difracción de rayos X, con los cuales se determina la estructura de proteínas.

\section{BIBLIOGRAFÍA}

[1] Shen, Y. X., Saboe, P. O., Sines, I. T., Erbakan, M., \& Kumar, M.. Biomimetic membranes: a review. Journal of Membrane Science, 454, 359-381, 2014.

[2] Whitford, D. Proteins: structure and function. John Wiley \& Sons, 2013.

[3] Darby, N. J., \& Creighton, T. E. Protein structure (pp. 1-41). Oxford, UK: IRL Press at Oxford University Press, 1993.

[4]http://www.rscb.org/pdb/static.do?p=general_information/ about_pdb/nature_of_3d_structural_dat.html
[5] Schnell, J. R., \& Chou, J. J. Structure and mechanism of the M2 proton channel of influenza A virus. Nature, 451(7178), 591-595, 2008.

[6] Maldonado, A., Urbach, W., Ober, R., \& Langevin, D. Swelling behavior and local topology of an L 3 (sponge) phase. Physical Review E, 54(2), 1774-1778, 1996.

[7] Maldonado, A., Ober, R., Gulik-Krzywicki, T., Urbach, W., \& Langevin, D. The sponge phase of a mixed surfactant system. Journal of colloid and interface science, 308(2), 485490, 2007.

[8] Ridell, A., Ekelund, K., Evertsson, H., \& Engström, S. On the water content of the solvent/monoolein/water sponge (L3) phase. Colloids and Surfaces A: Physicochemical and Engineering Aspects, 228(1), 17-24, 2003.

[9] Kulkarni, C. V., Wachter, W., Iglesias-Salto, G., Engelskirchen, S., \& Ahualli, S. Monoolein: a magic lipid?. Physical Chemistry Chemical Physics, 13(8), 3004-3021, 2011.

[10] Gambin, Y., Lopez-Esparza, R., Reffay, M., Sierecki, E., Gov, N. S., Genest, M., Urbach, W. Lateral mobility of proteins in liquid membranes revisited. Proceedings of the National Academy of Sciences of the United States of America, 103(7), 2098-2102, 2006.

[11] Maldonado, A., Urbach, W., \& Langevin, D. Surface selfdiffusion in L3 Phases. The Journal of Physical Chemistry B, 101(41), 8069-8073, 1997.

[12] Li, D., Shah, S. T., \& Caffrey, M. Host lipid and temperature as important screening variables for crystallizing integral membrane proteins in lipidic mesophases. Trials with diacylglycerol kinase. Crystal growth \& design, 13(7), 28462857, 2013.

[13] Caffrey, M. Crystallizing membrane proteins for structurefunction studies using lipidic mesophases. In Advancing Methods for Biomolecular Crystallography (pp. 33-46). Springer Netherlands, 2013.

[14] Caffrey, M., Li, D., \& Dukkipati, A. Membrane protein structure determination using crystallography and lipidic mesophases: recent advances and successes. Biochemistry, 51(32), 6266-6288, 2012.

[15] Joseph, J. S., Liu, W., Kunken, J., Weiss, T. M., Tsuruta, H., \& Cherezov, V. Characterization of lipid matrices for membrane protein crystallization by high-throughput small angle X-ray scattering. Methods, 55(4), 342-349, 2011.

[16] Johansson, L. C., Arnlund, D., White, T. A., Katona, G., DePonte, D. P., Weierstall, U., \& Schlichting, I. Lipidic phase membrane protein serial femtosecond crystallography. Nature Methods, 9(3), 263-265, 2012.

[17] Oka, T., \& Hojo, H. Single Crystallization of an Inverse Bicontinuous Cubic Phase of a Lipid. Langmuir, 30(28), 82538257,2014

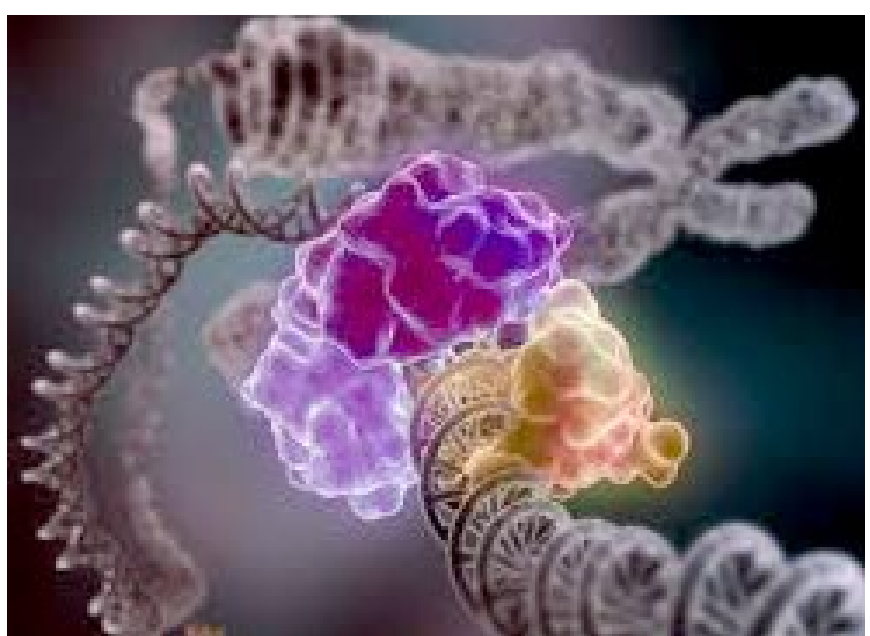

